\title{
STUDY ON HIGH FLUX ACCELERATOR BASED POSITRONS SOURCE *
}

\author{
J. Long, S. Chemerisov, W. Gai ,C. D. Jonah, W. Liu, H. Wang, \\ ANL, Argonne, IL 60439, U.S.
}

\begin{abstract}
Present accelerator-based slow-positron technology impinge the positrons coming out of a conversion target on a thin foil of metal (moderator) where the positrons are thermalized and then ejected from the moderator with the surface work energy of the material. This study represents a new direction in developing accelerator-based highintensity slow-positron sources. We investigate the use of RF fields to decelerate the positrons out of the target. Techniques, such as a Penning trap or moderation can then be used to get the needed thermal positrons with higher yield. Using a relatively simple beam-line scheme, our preliminary simulations showed that it could increase the final thermal positrons yield by more than 20 times. This suggests a promising future for this new method.
\end{abstract}

\section{INTRODUTION}

Positrons have tremendous potential in many fields of science, including chemistry, physics, materials science, surface science, biological sciences. However, the utilization of positrons in these fields of research has been strongly inhibited by the inability to get a sufficient flux of positrons to utilize proposed techniques such as positron microprobes, positron holography, or to carry out gravitation experiments with antimatter or to do antimatter chemistry[1]. Compared to a radioactiveisotope positron source, accelerator-based positron source can reach much higher intensities. These positrons are produced in the conversion target due to e+/e- pair process when bremsstrahlung photons interact with target atoms. But the spectrum of the positrons is not appropriate for the needed research. Instead of highenergy positrons (energies primarily greater than 150 $\mathrm{keV}$ ), one needs positrons at thermal energies or at welldefined energies in the range of $\mathrm{keV}$.

Present technology uses "moderation" to obtain thermal positrons. Positrons out of the target impinge on a material where they are slowed to thermal energies. These slowed positrons can then be ejected from the surface at a few volts, if the work function for the surface is appropriate. The efficiency of this process is on the order of $10^{-4}$ to $10^{-5}$ low energy positrons per incident positron [2]. Solid rare-gas moderators may increase this efficiency to greater than $10^{-2}$; however, these techniques are still in the early stages and are limited by the energy deposited[3]. To use solid rare gas moderator in an accelerator-based positron source, positrons would have to be separated from high energy electrons and X-rays.

A slow positron is generated when a positron is

\footnotetext{
*Work supported by DOE

"long@anl.gov
}

thermalized sufficiently close to the surface of the moderator so that the positron can diffuse to the surface without annihilating. Lower energy positrons have greater probability being stopped near the surface. Experimental data suggest that the moderation of a tungsten foil could reach 0.23 for $1-5 \mathrm{keV}$ positrons.[4] In addition, lower energy positrons will deposit less energy in the material and thus reduce radiation damage to single-crystal moderators or decrease thermal load on cryogenic moderators.

Another option is that it would be possible to trap and cool positrons in a trap, if the energy of the positrons can be decreased below $100 \mathrm{keV}$. (There are reports that the potential of the gate electrodes on a Penning trap may be as high as $100 \mathrm{kV}$ ). The positrons will be cooled in the trap by collision with one another and by cyclotron radiation.

Because the present yield of slow positrons from the moderation of high-energy positrons is so low, moderate success in decreasing the energy of positrons generated by high-energy accelerators could make substantial increase in the generation of slow positrons. Our first goal with this research is to explore the possibility of decelerating high-energy positrons using a relatively simple deceleration scheme. To match with the possible subsequent processes described above we set the goal for this research:

1. To decelerate a small fraction of the positrons to energies below $100 \mathrm{keV}$ and with relatively narrow $(<20 \mathrm{keV})$ energy spectrum so that the positrons can then be efficiently decelerated using electrostatic fields and then cooled to thermal energy in a positron trap.

2. To decrease a major fraction of the positrons to energies below $100-200 \mathrm{keV}$ and thus be able to more efficiently moderate these positrons.

All relevant parameters in our work are based on a $15 \mathrm{MeV}$ linac and other available equipment such as a 108 $\mathrm{MHz}$ RF source in the Chemistry Div. at Argonne National Lab. If possible, the future experimental work will be carried on this facility.

\section{BEAM-LINE DESIGN}

Our initial attempts to design the beam line encounter a significant problem. The positrons which are products of the electron-positron pair reaction have a large spread of transverse and longitudinal momenta and there is little correlation between the transverse and longitudinal momenta For this reason, it is difficult to define a reference particle. This limits the applicability of one- 
dimensional modelling and lots of simulations have had to be employed.

Figure 1 is the beam-line schematic that we used for simulation. Between the conversion target and RF cavity, there is an Adiabatic Matching Device (AMD), mainly used for making the transverse phase ellipse of the beam rotate 90 degree. The transverse momentum of the positrons' could be reduced after passing through the AMD.

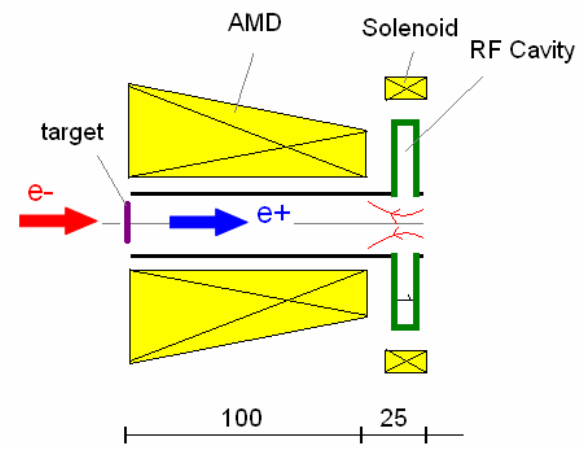

Figure 1: Schematic of the slow-positron beam-line design, cavity gap $=5 \mathrm{~cm}$, considering the fringe field, the total length of affected region along $\mathrm{z}$ is set $25 \mathrm{~cm}$.

In the $\mathrm{AMD}$, magnetic field along $\mathrm{z}$ axis decreases from 10000 Gauss to 720 gauss from entrance to exit (100 centimetres). The field in the AMD satisfies optimized design equation [5].

$$
B_{z}=\frac{10000}{1+0.129 \cdot z}
$$

The parameters of the AMD are optimized by adjusting the AMD length, field strength to get a small RMS (root mean square) positron divergence at the AMD while minimizing the loss of positrons. The maximum magnetic field is limited by available conventional magnets.

After the AMD, a pillbox cavity is used to decelerate the positrons. The axis size of the cavity is fixed and its radial size could be adjusted to get different resonant frequencies. The beam pipe radius in the simulation was 7 centimetres. The magnetic field around the drift space and the cavity is 720 gauss.

\section{SIMULATION AND RESULT}

A general charged-particle transport code, Parmela [6], is used to simulate positrons movement in the beamline. The initial positron parameters are from MonteCarlo simulation code EGSnrc[7] for $15 \mathrm{MeV}$ electrons impinging on a $0.2 \mathrm{~cm}$ tungsten target.

\section{Transverse Ellipse Transformation}

For positron deceleration, the AMD is very important. Figure 2 shows the transverse phase ellipse transformation of the beam.

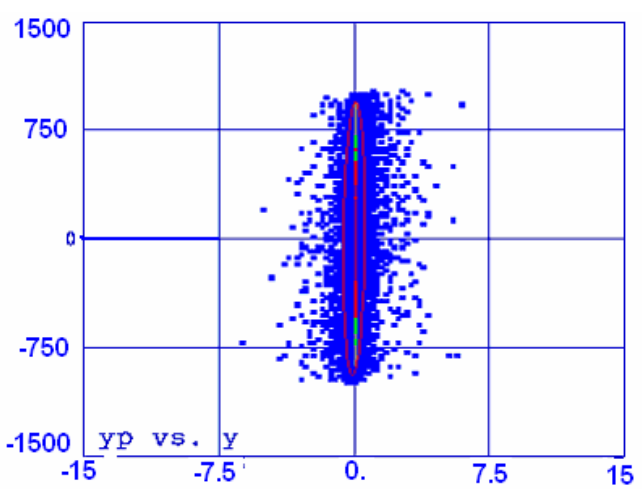

(a)

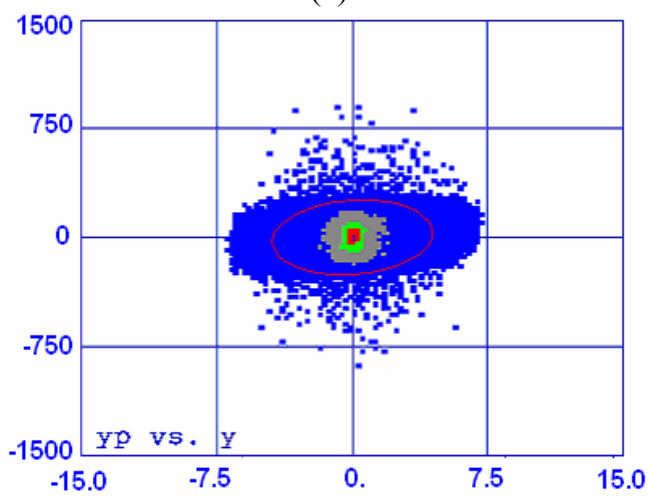

(b)

Figure 2: (a) transverse phase ellipse of the beam at the AMD entrance, (b) transverse phase ellipse of the beam at the exit; horizontal coordinator is $\mathrm{x}$ axis in $\mathrm{cm}$, vertical coordinator is $\mathrm{x}$ prime $(\mathrm{Px} / \mathrm{Pz})$ in $\mathrm{mrad}$.

The spectrum of the particles' longitudinal velocity before and after AMD are presented in Figure 3, to give a better understanding of the function of AMD

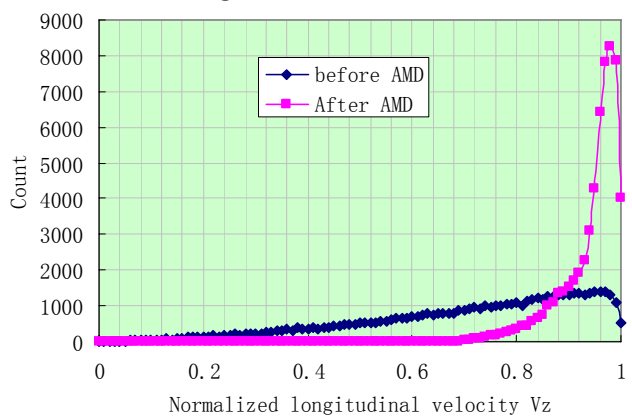

Figure 3: comparison of longitudinal velocity spectrum before and after AMD, Here, the velocity is normalized by c (light speed in vacuum) and each bin $=0.01 \mathrm{c}$

\section{Deceleration Results}

We have simulated two different cases. Case 1 is only $108 \mathrm{MHz}$ RF field existing in the cavity; Case 2 attempts to put both $108 \mathrm{MHz}$ and $216 \mathrm{MHz}$ in the cavity to see how much improvement could got. By changing amplitudes and initial phases of its rf field, we could get different result. We try to slow down positrons as much we can. The best results are showed in Fig.4, the histograms are plotted with $10 \mathrm{keV}$ bin. For case 1, the peak value is around 873 positrons out of 59034 within 
[80keV,100keV], which means about $1.47 \%$ of total positrons in the band. For case 2, the peak location shifts to $[40 \mathrm{keV}, 60 \mathrm{keV}]$ while value raised to $1.6 \%$ of total positrons.

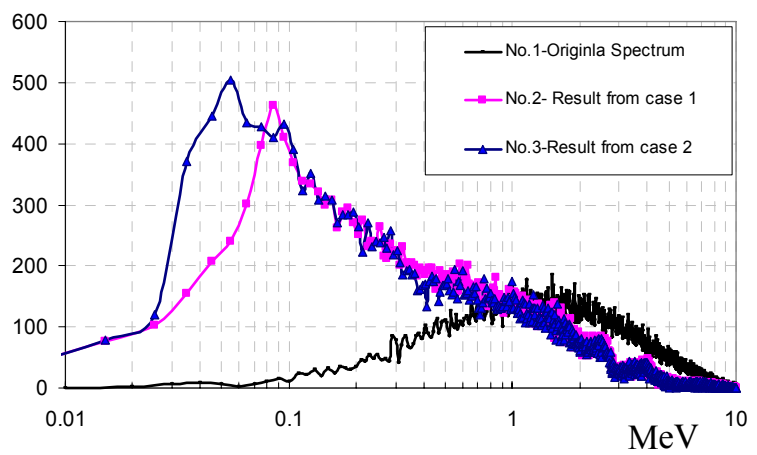

Figure 4: the energy spectrums for different cases

In both cases, the average axial electrical fields are less than $5 \mathrm{MV} / \mathrm{m}$ in the cavity. It seems feasible in practice.

\section{Moderation}

Moderation efficiencies of tungsten foil for different energy positrons were calculated using EGSnrc for positrons from $20 \mathrm{keV}$ to $2 \mathrm{MeV}$. It was assumed that the moderation efficiency would be proportional to the number of positrons whose energies were reduced below $2 \mathrm{keV}$ within a region that is $10^{-6} \mathrm{M}$ of the surface (details of these calculations will be published later). Using these data and the spectra in figure 4, both reflection moderation efficiency and transmission moderation efficiency are given in figure 5. These values are normalized by the corresponding values of original positrons beam.

For case 1, sole RF field in the cavity, the largest gain is around 20 times for reflection yield while 30 for transmission yield. For case 2, both yields increase over $50 \%$. If we look back to fig. 4 , by comparing the spectrums 2 and 3, it seems that within keV range, lower positions energy could raise yield efficiently. So we shifted the input spectrum for case $320 \mathrm{keV}$ lower. That, in practice, would correspond to a $20 \mathrm{kV}$ DC gap downstream from the RF cavity.

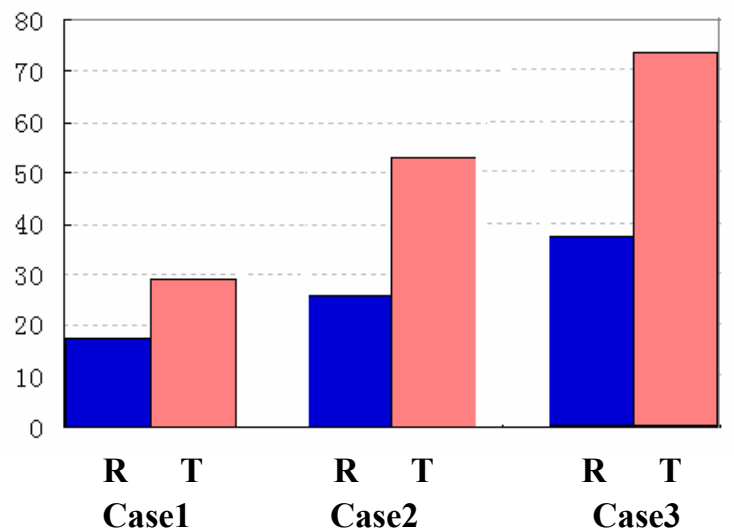

Figure 5: Histogram of moderation efficiency, here $\mathrm{R}$ means reflection and $\mathrm{T}$ means transmission
Result of case 2 and 3 show even a small shift in energy substantially increased both kinds of yields. Thus we believe that an increase in yield of slow positrons by two-orders-of-magnitude is possible.

\section{CONCLUSIONS}

In this work, we have shown that it is possible to use $\mathrm{RF}$ fields and accelerator techniques to increase the yield of slow positrons from high-energy positrons. These calculations suggest it is possible to increase the yields 2orders-of-magnitude greater than what is conventionally available by decreasing the energy and increasing the positron thermalization yield in the moderator or decreasing the energy and energy spread and injecting into a trap. However, because most of the positrons are still not utilized, there is still considerable opportunity to increase the yields of slow positrons.

\section{REFERENCES}

[1] P.J.Schultz and K.G.Lynn, Rev.Mod.Phys.60 (1983) 701

[2] A.Vehanen,K.G.Lynn,P.J.Schultz, Appl.Phys.A 32 (1983) 163

[3] A.P. Mills and E. M. Gullikson, Appl. Phys. Lett. 49 (1988) 1121.

[4] G. Kogel, G. Dollinger, Applied Surface Science, 252 (2006) 3111.

[5] R.Chehab, IEEE Transactions on Nuclear Science, 30-4 (1983) 2850.

[6] Lloyd Young and James Billen, "The Particle Tracking Code PARMELA", PAC'2003, Portland, May 2003, p3521.

[7] I. Kawrakow, Med. Phys. 27 (2000) 485. 